\title{
Proteomic analysis reveals that MAEL, a component of nuage, interacts with stress granule proteins in cancer cells
}

\author{
LIQIN YUAN ${ }^{1}$, YUZHONG XIAO ${ }^{2}$, QIUZHI ZHOU ${ }^{2}$, DONGMEI YUAN ${ }^{2}$, \\ BAIPING WU ${ }^{1}$, GANNONG CHEN $^{1}$ and JIANLIN ZHOU ${ }^{2}$ \\ ${ }^{1}$ Department of General Surgery, The Second Xiangya Hospital, Central South University, \\ Changsha, Hunan 410011; ${ }^{2}$ Key Laboratory of Protein Chemistry and Developmental Biology of the Ministry of Education, \\ College of Life Science, Hunan Normal University, Changsha, Hunan 410081, P.R. China
}

Received September 24, 2013; Accepted October 21, 2013

DOI: $10.3892 /$ or.2013.2836

\begin{abstract}
The Maelstrom (MAEL) gene is a cancer-testis (or cancer-germline) gene, which is predominantly expressed in germline cells under normal conditions, but is aberrantly expressed in a range of human cancer cells. In germline cells, MAEL is found predominantly in the nuage, where it plays an essential role in piRNA biogenesis and piRNA-mediated silencing of transposons. However, the role of MAEL in cancer has not been elucidated. We performed immunoprecipitation and Nano-LC-MS/MS analysis to investigate the interactome of MAEL, and identified 14 components of stress granules (SGs) as potential binding partners of MAEL in MDA-MB-231 human breast cancer and SW480 colorectal cancer cells. The interactions between MAEL and 8 of these SG components (PABPC1, YBX1, KHSRP, SYNCRIP, DDX39, ELAV1, EIF4A1 and EIF3F) were confirmed by anti-tag immunoprecipitation. Immunofluorescence analysis showed that MAEL co-localizes with the SG marker PABPC1 in SGs during oxidative stress. Nuages and SGs are the cytoplasmic RNA granules of germline cells and stressed somatic cells, respectively, and both serve as a platform for small RNA-mediated gene silencing. It is, therefore, suggested that MAEL may be involved in miRNAmediated gene silencing in SGs, as it does in the nuage. This finding should be valuable toward understanding the function of MAEL in carcinogenesis.
\end{abstract}

Correspondence to: Professor Gannong Chen, Department of General Surgery, The Second Xiangya Hospital, Central South University, Changsha, Hunan 410011, P.R. China

E-mail: cgn01hn@163.com

Professor Jianlin Zhou, Key Laboratory of Protein Chemistry and Developmental Biology of the Ministry of Education, College of Life Science, Hunan Normal University, Changsha, Hunan 410081, P.R. China

E-mail: jlzhou@hunnu.edu.cn

Key words: MAEL, cancer-testis gene, nuage, stress granule, proteomic analysis

\section{Introduction}

The Maelstrom (MAEL) gene was first identified in Drosophila (1). It plays a role in the establishment of oocyte polarity by acting in the positioning of mRNA and the microtubuleorganizing center (MTOC) in early oocytes $(1,2)$. Drosophila maelstrom protein co-localizes with Vasa and Aubergine in the nuage (3), a germline-unique perinuclear structure that serves as a platform for PIWI-interacting RNA (piRNA) biogenesis and piRNA-dependent silencing of transposons and some other harmful selfish elements $(4,5)$. The mouse homolog of MAEL is also found to localize in the nuage $(6,7)$ and is essential for spermatogenesis and transposon repression (7). Further study by Aravin et al (8) demonstrated that MAEL co-localizes with MIWI2/PIWIL4 in a type of nuage named piP-body, which harbors both piRNA pathway proteins (MIWI2, TDRD9 and MAEL) and P-body (processing body) components (DDX6, DCP1a, XRN1 and GW182), and that loss of MAEL disrupts normal MIWI2 localization and piRNA production leading to transposon activation. In rat spermatogenic cells, MAEL protein is detected in all types of nuage except the cluster of $30-\mathrm{nm}$ particles (i.e. 70 - to $90-\mathrm{nm}$ particles, satellite body, intermitochondrial cement, cluster of 60- to $90-\mathrm{nm}$ particles, chromatoid body) (9). MAEL protein is also found in nonnuage compartments $(10)$ and the nucleus $(3,9)$. In the nucleus, MAEL binds the miR-7 promoter and represses its expression (11).

Our previous study demonstrated that MAEL is a cancer-testis (CT) gene (12). The CT genes, also known as cancer-germline (CG) genes, are predominantly expressed in germ cells of the testis or/and ovary and have no or little expression in somatic adult tissues, but are aberrantly expressed in various types of cancers $(13,14)$. We found that the human MAEL gene is only expressed in germ cells of the testis among normal tissues, but is expressed in various human cancer cell lines such as breast cancer MDA-MB-231 cells and colorectal cancer SW480 cells. Like most of the CT genes $(15,16)$, the expression of the MAEL gene is activated by hypomethylation in cancer (12). Kim et al (17) analyzed the methylation profile of $27,578 \mathrm{CpG}$ sites spanning $>14,000$ genes in colorectal cancer and adjacent normal mucosa, and found that among the genes tested, MAEL and SFT2D3 
Table I. Primers used for amplifying the coding region of the genes.

\begin{tabular}{lll}
\hline Gene name & \multicolumn{1}{c}{ Sequence $\left(5^{\prime} \rightarrow 3^{\prime}\right)$} & Restriction site \\
\hline MAEL-F & GAATTCCCATGCCGAACCGTAAGG & EcoRI \\
MAEL-R & GGTACCGGGCCTGTTACTGTTTTCAGAA & $K p n I$ \\
DDX39-F & GAATTCTCATGGCAGAACAGGATGTG & EcoRI \\
DDX39-R & CTCGAGTGGTGGTTACCGGCTCTG & XhoI \\
EIF4A1-F & GAATTCTCATGTCTGCGAGCCAGG & EcoRI \\
EIF4A1-R & GTCGACGCTGGGTGGCAGGACAG & SalI \\
EIF3F-F & GAATTCACAAGATGGCCACACCG & EcoRI \\
EIF3F-R & CTCGAGCCATTCACAGGTTTACAAGTTT & XhoI \\
ELAV1-F & GTCGACAATGTCTAATGGTTATGAAGACC & SalI \\
ELAV1-R & GGTACCGCATGAGCGAGTTATTTGTG & $K p n I$ \\
PABPC1-F & GTCGACCGAGATGAACCCCAGTGC & SalI \\
PABPC1-R & GGTACCTTTAAACAGTTGGAACACCG & $K p n I$ \\
SYNCRIP-F & GTCGACTGGAAACATGGCTACAGAACA & SalI \\
SYNCRIP-R & GGTACCCTACTTCCACTGTTGCCCAA & $K p n I$ \\
KHSRP-F & GAATTCCCATGTCGGACTACAGCAC & $E c o R I$ \\
KHSRP-R & GGTACCGATTCATTGAGCCTGCTGC & $K p n I$ \\
\hline
\end{tabular}

showed the lowest methylation level in tumor tissues when compared to the normal mucosa (17). However, the precise subcellular localization and function of MAEL in cancer cells are not clear.

In the present study, we identified the interacting partners of MAEL in breast cancer MDA-MB-231 and colorectal cancer SW480 cells using mass spectrometric method, and demonstrated that MAEL localizes in stress granules (SGs) in cancer cells and interacts with several components of SGs.

\section{Materials and methods}

Plasmids. The Myc-tagged YBX1 expression plasmid pDESTmycYBX1 [Addgene plasmid 19878, deposited by Landthaler (18)] was obtained from Addgene (Cambridge, MA, USA). The other expression plasmids (pHA-MAEL, pMyc-DDX39, pMyc-EIF4A1, pMyc-EIF3F, pMyc-ELAV1, pMyc-PABPC1, pMyc-SYNCRIP and pMyc-KHSRP) were constructed as follows. The coding sequence of each gene was amplified from the first strand cDNA of MDA-MB-231 or SW480 cells with the primers described in Table I. The amplified products were cloned into the pMD18-T vector (Takara, Dalian, China) by TA cloning method, and subcloned into the pCMV-HA or pCMV-Myc vector (Clontech Laboratories, Inc., Mountain View, CA, USA) with restriction enzyme sites in the primer (Table I).

Immunoprecipitation (IP) of the MAEL protein complex. The human breast cancer MDA-MB-231 and colorectal cancer SW480 cells [American Tissue Culture Collection (ATCC), Manassas, VA, USA] were cultured in L-15 media that was supplemented with glutamine, antibiotics and $10 \%$ fetal bovine serum (FBS) at $37^{\circ} \mathrm{C}$ in $5 \% \mathrm{CO}_{2}$. The cells were washed with phosphate-buffered saline (PBS) and incubated with $0.5 \mathrm{mM}$ cross-linker DSP (Pierce, Rockford, IL, USA) for $30 \mathrm{~min}$. After cross-linking, cells were sequentially washed with PBS,
PBS containing $25 \mathrm{mM}$ Tris- $\mathrm{HCl}$ buffer and PBS. Then, cells were lysed in RIPA buffer by sonication, and subjected to centrifugation at $12,000 \mathrm{x}$ g for $15 \mathrm{~min}$. The supernatants were transferred to a new tube for immunoprecipitation.

Immunoprecipitation was performed as described by Lin et al (19). In each 1.5-ml tube, $2.5 \mathrm{mg}$ of lysates was mixed with $2 \mu \mathrm{g}$ of polyclonal antibody against MAEL or preimmune rabbit IgG (Signalway Antibody Co., Ltd., Nanjing, China), and incubated overnight at $4^{\circ} \mathrm{C}$ with gentle shaking. Immune complexes were collected on protein $\mathrm{A} / \mathrm{G}$ agarose beads and washed three times to remove non-specific binding using the same buffer as for the cell lyses. Proteins were eluted from the beads by boiling the beads in loading buffer for $10 \mathrm{~min}$, and were then separated on SDS-PAGE gel and visualized with silver staining.

Nano-LC-MS/MS. Each excised protein gel band was destained and digested with trypsin as described by Lin et al (19). Nano-LC MS/MS experiment was performed on an HPLC system composed of two LC-20AD nano-flow LC pumps, an SIL-20AC autosampler and an LC-20AB micro-flow LC pump (Shimadzu, Tokyo, Japan) connected to an LTQ Orbitrap mass spectrometer (Thermo Fisher Scientific, San Jose, CA, USA). Sample was loaded on a CapTrap column $(0.5 \times 2 \mathrm{~mm}$; Michrom Bioresources, Inc., Auburn, CA, USA) for $6 \mathrm{~min}$ at a flow rate of $25 \mu \mathrm{l} / \mathrm{min}$. The sample was subsequently separated by a $\mathrm{C} 18$ reverse-phase column $(0.10$ x $150 \mathrm{~mm}$, packed with $3 \mu \mathrm{m}$ Magic C18AQ particles; Michrom Bioresources) at a flow rate of $500 \mathrm{nl} / \mathrm{min}$. The mobile phases were $2 \%$ acetonitrile with $0.1 \%$ formic acid (phase A and the loading phase) and $95 \%$ acenitrile with $0.1 \%$ formic acid (phase B). To achieve proper separation, a 60-min linear gradient from 0 to $80 \%$ phase B was employed. The separated sample was introduced into the mass spectrometer via an Advance $30-\mu \mathrm{m}$ silica tip (Michrom Bioresources). The spray voltage was set at $1.6 \mathrm{kV}$ and the heated capillary at $180^{\circ} \mathrm{C}$. The mass 
spectrometer was operated in the data-dependent mode, and each cycle of duty consisted of one full-MS survey scan at the mass range 385-2000 Da with resolution power of 100,000 using the Orbitrap section, followed by MS/MS experiments for 10 strongest peaks using the LTQ section. The AGC expectation during full-MS and MS/MS were 1,000,000 and 10,000 , respectively. Peptides were fragmented in the LTQ section using collision-induced dissociation with helium and the normalized collision energy value set at $35 \%$. Previously fragmented peptides were excluded for $60 \mathrm{sec}$.

Database search. Tandem mass spectra were extracted by BioWorks version 3.3.1 SP1 (Thermo Fisher Scientific). All MS/MS samples were analyzed using Sequest (Thermo Fisher Scientific; version 28). Sequest was set up to search the human proteome database from UniProt release 2010_08 (downloaded from the official website of UniProtKB following this link: http://www.uniprot.org/uniprot/?query=organism:960 6+keyword: $181 \&$ format $=* \&$ compress $=y e s$, downloaded at 2010-07-13) assuming the digestion enzyme trypsin. Sequest was searched with a fragment ion mass tolerance of $1.00 \mathrm{Da}$ and a parent ion tolerance of $20 \mathrm{ppm}$. Oxidation of methionine $(+15.99492 \mathrm{Da})$ and acetylation of lysine $(+42.01057 \mathrm{Da})$ were specified as variable modifications. Trans-Proteomic Pipeline (20) was used to validate MS/MS based peptide and protein identifications. Peptide probability was specified by the Peptide Prophet algorithm (21). Protein probabilities were assigned by the Protein Prophet algorithm (22). Proteins that contained similar peptides and could not be differentiated based on MS/MS analysis alone were grouped to satisfy the principles of parsimony.

Gene Ontology analysis. Gene Ontology (GO) enrichment analysis was performed using the Database for Annotation, Visualization and Integrated Discovery (DAVID) functional annotation tool (http://david.abcc.ncifcrf.gov) (23). UniProt accession numbers of MAEL-associated proteins were submitted to DAVID system and categorized based on biological process (BP), molecular function (MF) and cellular component (CC) using the software.

Anti-tag co-immunoprecipitation. Transfection and co-IP were performed as previously described (24). Hek293 cells were co-transfected with HA-tagged MAEL expression plasmids and expression plasmids of each Myc-tagged SG gene. At $24 \mathrm{~h}$ after transfection, cell lysates were prepared and immunoprecipitated with rabbit anti-HA polyclonal antibody or preimmune rabbit $\mathrm{IgG}$, and the precipitated protein was detected by western blot analysis using the anti-Myc monoclonal antibody. The anti-HA, anti-Myc and IgG antibodies were purchased from Santa Cruz Biotechnology (Santa Cruz, CA, USA).

Immunofluorescence staining of SGs. SW480 cells were grown on glass coverslips, and treated with $0.5 \mathrm{mM}$ hydrogen peroxide $\left(\mathrm{H}_{2} \mathrm{O}_{2}\right)$ for $1 \mathrm{~h}$ to induce the formation of SGs. Treated cells and control cells were fixed with $4 \%$ paraformaldehyde and permeabilized in $0.1 \%$ Triton X-100, respectively. To reduce the unspecific binding, the cells were incubated in $1 \%$ BSA overnight the $4^{\circ} \mathrm{C}$. Then, cells were incubated with anti-MAEL
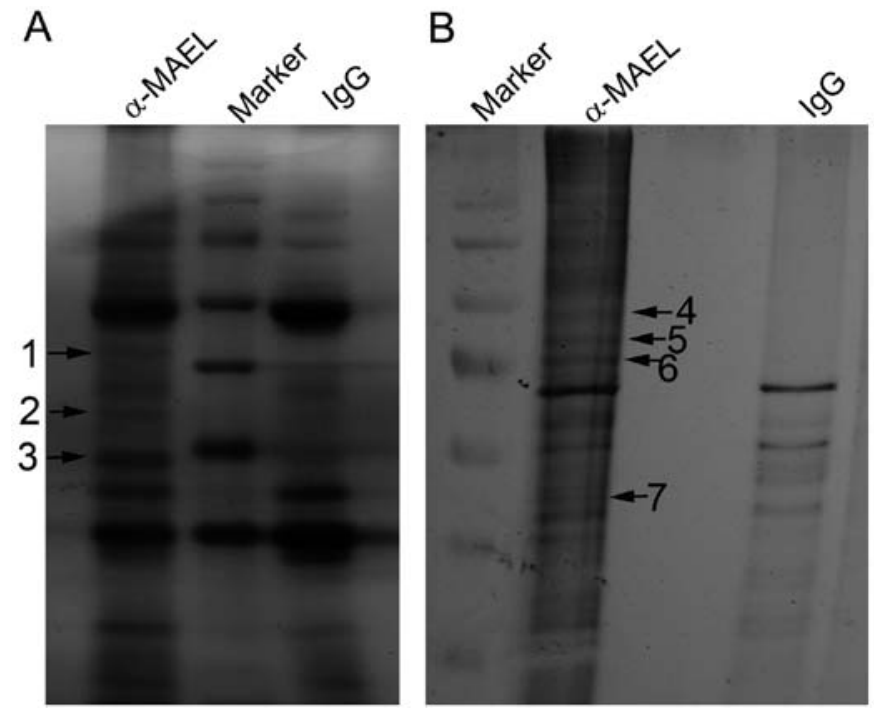

Figure 1. Silver-stained SDS-PAGE gel of the immunoprecipitates. The lysates $(2.5 \mathrm{mg}$ ) of (A) MDA-MB-231 or (B) SW480 cells were mixed with $2 \mu \mathrm{g}$ of anti-MAEL antibody or $\mathrm{IgG}$, and incubated overnight at $4^{\circ} \mathrm{C}$ with gentle shaking. Immune complexes were separated on SDS-PAGE gel and visualized with silver stain. The protein bands which only existed in the complexes of the anti-MAEL antibody are indicated by arrows.

polyclonal antibody and anti-PABPC1 monoclonal antibody (Pierce, Rockford, IL, USA) for $2 \mathrm{~h}$ at room temperature, followed by incubation with Texas Red-conjugated anti-rabbit IgG (red) and FITC-conjugated anti-mouse IgG (green) for $1 \mathrm{~h}$. The nuclei were labeled by DAPI. Images were obtained using a Zeiss LSM 510 confocal microscope (Carl Zeiss Imaging, Oberkochen, Germany). Texas Red-conjugated anti-rabbit IgG and FITC-conjugated anti-mouse IgG were purchased from Santa Cruz Biotechnology.

\section{Results}

RNA-binding proteins are enriched in the MAEL protein complex. To identify the potential interacting partners of MAEL protein, we performed the co-IP experiments to isolate the MAEL complex in human breast cancer MDA-MB-231 and colorectal cancer SW480 cells. The immune complexes of the anti-MAEL antibody and $\operatorname{IgG}$ were resolved on SDS-PAGE gel followed by silver staining. The protein bands which only existed in the complex of the anti-MAEL antibody were excised (Fig. 1), digested with trypsin and analyzed by Nano-LC MS/MS method. All MS/MS samples were analyzed using Sequest. According to the database search results, we identified 178 non-redundant proteins in the three bands from MDA-MB-231 cells and 167 proteins in the four bands from SW480 cells. Among these proteins, 78 proteins were common to both MDA-MB-231 and SW480 cells.

To find whether these 78 proteins share some particular features, we performed GO enrichment analysis with DAVID (23). Table II shows the top 5 significantly enriched terms in the MAEL-associated proteins. In the molecular function (MF) category, the RNA binding term is the most significant term (with the lowest P-value) and contains the largest number of proteins $(32.1 \%$ of the 77 proteins assigned 
Table II. Top 5 significantly enriched GO terms in the MAEL-associated proteins.

\begin{tabular}{|c|c|c|c|c|}
\hline Category & Term & Count & Percentage & Value \\
\hline \multirow[t]{5}{*}{ MF } & RNA binding & 25 & 32.1 & $1.12 \mathrm{E}-12$ \\
\hline & Structural constituent of the cytoskeleton & 10 & 12.8 & $4.04 \mathrm{E}-10$ \\
\hline & Structural molecule activity & 15 & 19.2 & $1.57 \mathrm{E}-05$ \\
\hline & Transcription corepressor activity & 6 & 7.7 & $1.60 \mathrm{E}-03$ \\
\hline & NAD or NADH binding & 4 & 5.1 & $2.60 \mathrm{E}-03$ \\
\hline \multirow[t]{5}{*}{$\mathrm{CC}$} & Non-membrane-bound organelle & 38 & 48.7 & 8.98E-10 \\
\hline & Ribonucleoprotein complex & 18 & 23.1 & $1.28 \mathrm{E}-09$ \\
\hline & Spliceosome & 11 & 14.1 & $1.98 \mathrm{E}-09$ \\
\hline & Intermediate filament & 10 & 12.8 & $5.62 \mathrm{E}-07$ \\
\hline & Nuclear lumen & 23 & 29.5 & 4.10E-06 \\
\hline \multirow[t]{5}{*}{$\mathrm{BP}$} & RNA splicing & 17 & 21.8 & 2.63E-12 \\
\hline & Chromatin assembly & 7 & 8.9 & $6.84 \mathrm{E}-06$ \\
\hline & Macromolecular complex assembly & 11 & 14.1 & $9.58 \mathrm{E}-06$ \\
\hline & Ectoderm development & 8 & 10.3 & $1.14 \mathrm{E}-04$ \\
\hline & Intermediate filament-based process & 4 & 5.1 & 2.34E-04 \\
\hline
\end{tabular}

MF, molecular function; $\mathrm{CC}$, cellular component; BP, biological process.

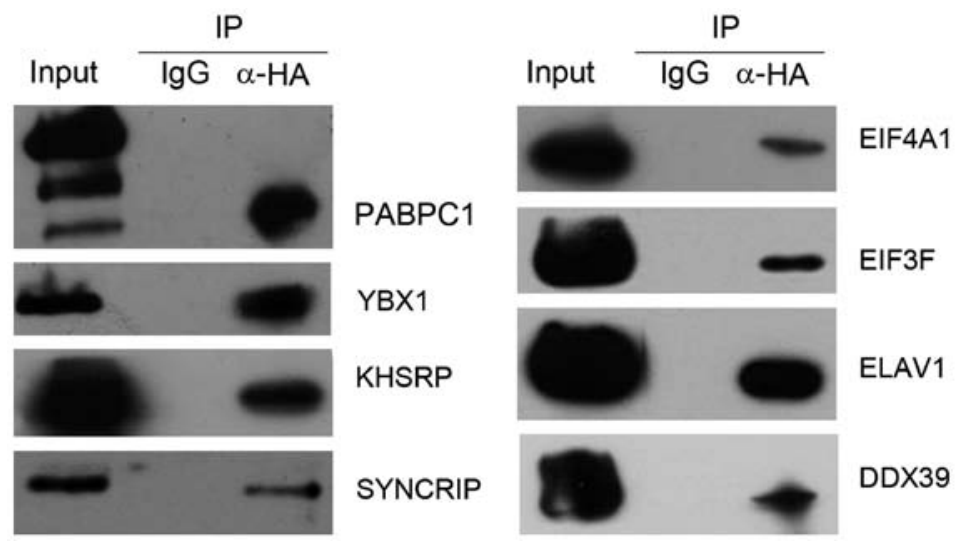

Figure 2. Interaction between MAEL and SG proteins as detected by anti-tag co-immunoprecipitation (IP). Hek293 cells were transfected with HA-tagged MAEL expression plasmids and expression plasmid of each Myc-tagged SG gene. At $24 \mathrm{~h}$ after transfection, cell lysates were prepared and immunoprecipitated with rabbit anti-HA polyclonal antibody or preimmune rabbit IgG, and the precipitated protein was detected by western blot analysis using the anti-Myc monoclonal antibody.

to MF category). In the cellular component (CC) category, the term with the lowest P-value was the non-membranebounded organelle, and that with the second lowest P-value was the ribonucleoprotein complex term. Additionally, a large number of MAEL-associated proteins were enriched in the nuclear lumen, consisting of 23 proteins $(29.5 \%$ of the 70 proteins assigned to $\mathrm{CC}$ category). In the biological process (BP) category, the top three terms with the lowest P-value were RNA process, chromatin assembly and macromolecular complex assembly. When considering all three categories, we found that most MAEL-associated proteins were RNA-binding proteins existing in the ribonucleoprotein complex.

MAEL interacts with stress granule proteins. MAEL protein is a component of nuage $(3,6,7)$, a germline-unique ribonu- cleoprotein complex particle. According to the above results (Table II), MAEL associates with RNA-binding proteins, possibly a component of the ribonucleoprotein complex incancer cells. However, we did not find other nuage components in the MAEL-associated proteins. Notably, the MAEL-associated proteins contained 14 components of SGs: PABPC1 (25), FUBP1/FBP1 (26), KHSRP/FBP2 (26), YBX1/YB-1 (27), SYNCRIP/hnRNP Q (28), EIF3F (29), EIF4A1 (30), EIF4B (31), ELAVL1 (32), HNRNPA1 (33), HNRNPA2B1 (34), DDX1 (27), DDX3X (35) and DDX39 (36). Among these, 8 proteins (PABPC1, FUBP1, KHSRP, YBX1, SYNCRIP, EIF4B, HNRNPA1 and HNRNPA2B1) were identified from both MDA-MB-231 and SW480 cells (Table III); 6 proteins were found only in MDA-MB-231 (EIF4A1, EIF3F and DDX3X) or SW480 cells (DDX39, ELAVL1 and DDX1) (data not shown). 

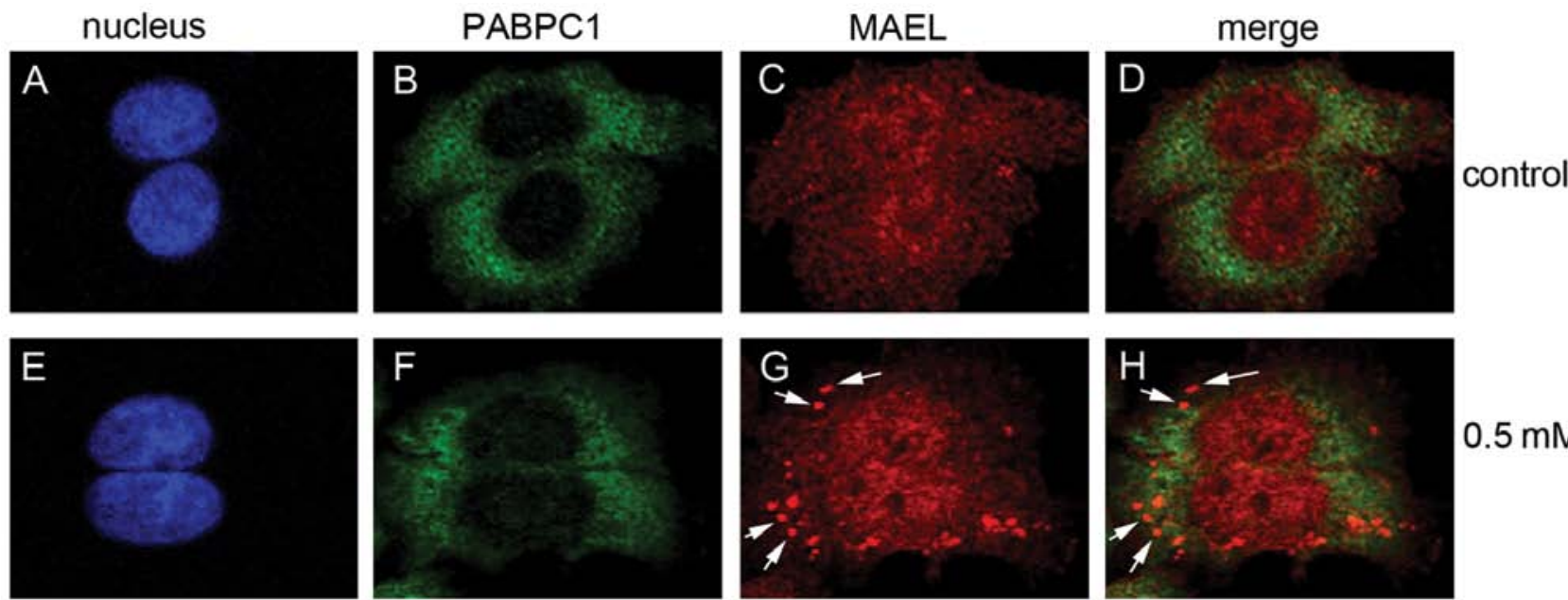

$0.5 \mathrm{mM} \mathrm{H}_{2} \mathrm{O}_{2}$

Figure 3. Co-localization of MAEL protein and PABPC1 in SGs. SW480 cells were treated with $0.5 \mathrm{mM} \mathrm{H}_{2} \mathrm{O}_{2}$ for $1 \mathrm{~h}$ to induce the formation of SGs. Treated cells (E-H) and control cells (A-D) were fixed and sequentially incubated with primary and secondary antibodies. The rabbit anti-MAEL polyclonal antibody and Texas Red-conjugated anti-rabbit IgG (red) were used to detect MAEL, whereas murine anti-PABPC1 monoclonal antibody and FITC-conjugated antimouse IgG (green) were used to detect PABPC1. The nuclei were labeled by DAPI. Yellow in the merged image represents co-localization of MAEL and PABPC1. The arrows indicate representative SGs.

Then, 8 (PABPC1, KHSRP, YBX1, SYNCRIP, EIF4A1, DDX39, ELAVL1 and EIF3F) of the identified SG proteins were selected for further confirmation of their interactions with MAEL protein by co-IP experiments. The coding regions of these 8 genes were cloned into mammalian expression vector $\mathrm{pCMV-Myc} \mathrm{for} \mathrm{constructing} \mathrm{Myc-tagged} \mathrm{expression}$ plasmids. The Myc-tagged expression plasmid of each SG protein was co-transfected with HA-tagged MAEL expression plasmid into Hek293 cells, respectively. The anti-tag immunoprecipitation assays showed that all these $8 \mathrm{SG}$ proteins were able to interact with the MAEL protein (Fig. 2).

MAEL localizes to SGs. As stated above, MAEL protein interacts with SG proteins. Therefore, we performed immunofluorescence analysis to investigate whether the MAEL protein localizes in SGs. In the untreated SW480 cells, MAEL proteins were distributed homogeneously in both the cytoplasm and the nucleus (Fig. 3). After induction with $\mathrm{H}_{2} \mathrm{O}_{2}$, some cytoplasmic MAEL proteins displayed a distribution pattern of distinct speckles, and co-localization with SG marker PABPC1 (25) in the speckle (Fig. 3), suggesting that MAEL proteins localize to SGs. Strangely, after treatment with $\mathrm{H}_{2} \mathrm{O}_{2}$, PABPC1 did not condense into apparent speckles as it did in the cells treated with arsenite (37).

\section{Discussion}

Our previous study demonstrated that the human MAEL gene is only expressed in the spermatocytes and spermatids of the testis under normal condition, but is aberrantly expressed in various types of human cancer cells (12). The role of MAEL in spermatogenesis has been studied thoroughly, but no investigation on its function in cancer has been performed. In the present study, we identified 178 MAEL-associated proteins in breast cancer MDA-MB-231 cells and 167 in colorectal cancer SW480 cells by immunoprecipitation and NanoLC-MS/MS analysis. In these MAEL-associated proteins, we found 14 components of SGs, but none of the nuage. The protein interactions between MAEL and these stress granule proteins were confirmed by anti-tag immunoprecipitation. Immunofluorescence analysis showed that MAEL co-localizes with PABPC1 in SGs during oxidative stress. This is not surprising, given that both SGs and nuage are cytoplasmic ribonucleoprotein complex particles (RNA granules). Nuage is a germline-unique structure (4), whereas SGs are somatic RNA granules and are assembled when cells are exposed to stress $(25,38,39)$. However, both nuage and SGs have similar composition, and serve as sites for small RNA-mediated gene silencing (40). MAEL has been found predominantly in the nuage of germline cells, where it plays an indispensable role in piRNA biogenesis and piRNA-mediated silencing of transposons $(3,6,7)$. Therefore, we hypothesized that MAEL plays a role in miRNA-mediated gene silencing in SGs, as it does in the nuage (7).

Additionally, we demonstrated that in cancer cells, the MAEL protein is distributed in both the cytoplasm and the nucleus, similar to its localization in germline cells $(3,7)$. In the nucleus, MAEL functions as a regulator of gene expression. For example, mouse MAEL was found to interact with chromatin-remodeling factors SNF5 and SIN3B in the testis (6); Drosophila MAEL represses the transcription of miR-7 (11). In accordance with the nuclear function of MAEL in germline cells, Gene Ontology annotation showed that some MAEL-associated proteins in cancer cells are involved in chromatin remodeling and transcription repression.

Our results suggest that MAEL plays a similar role in germline and cancer cells, similar to most CT genes. For example, GAGE functions as a regulator of chromatin reorganization in both germ and cancer cells (41). To date, 156 families of CT genes have been collected in the CT database (http://www.cta.Incc.br). The discovery of CT genes led to the hypothesis that gametogenesis and carcinogenesis share a similar mechanism; the expression of germline genes in cancer reflects the activation of the silenced gametogen- 


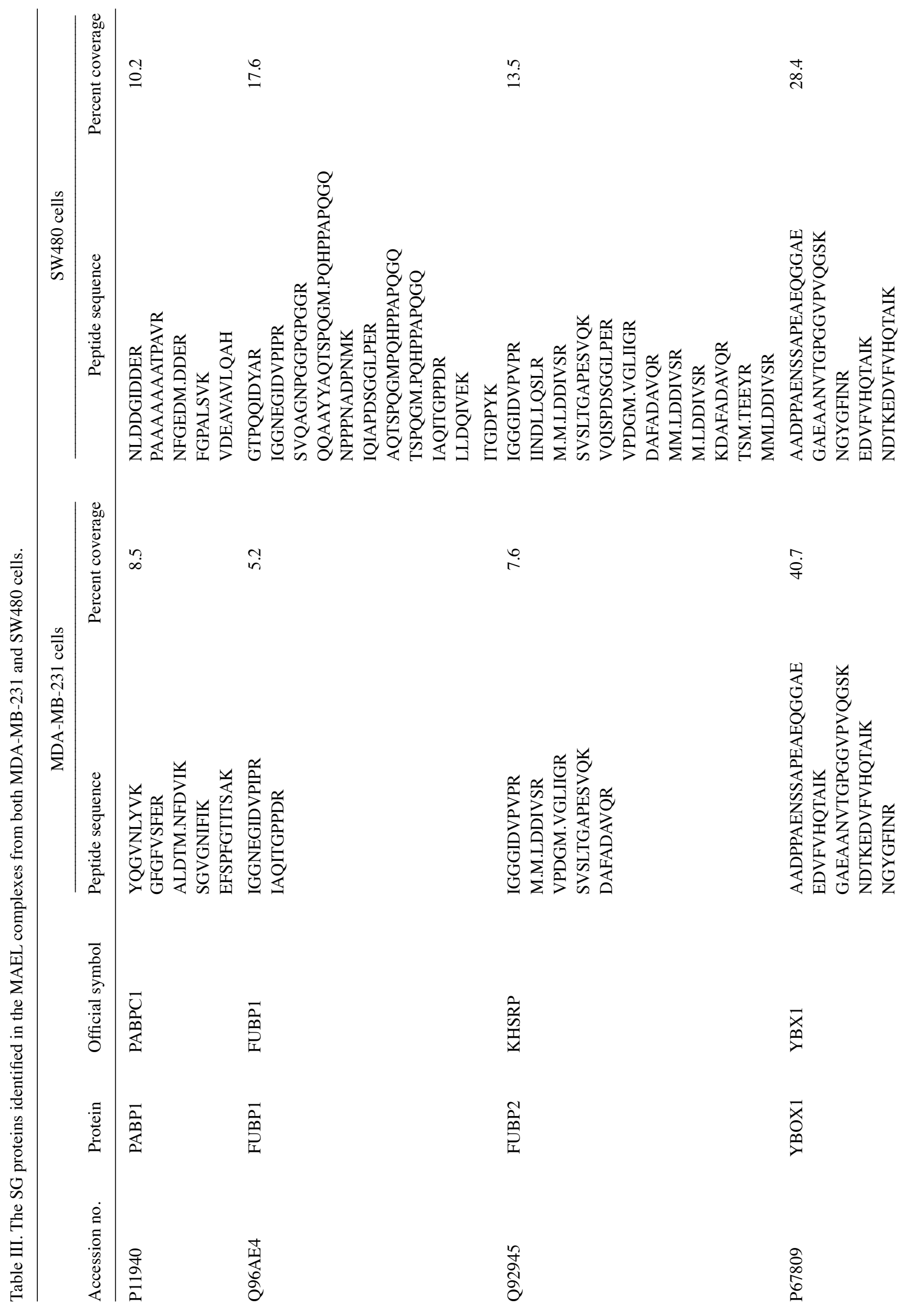




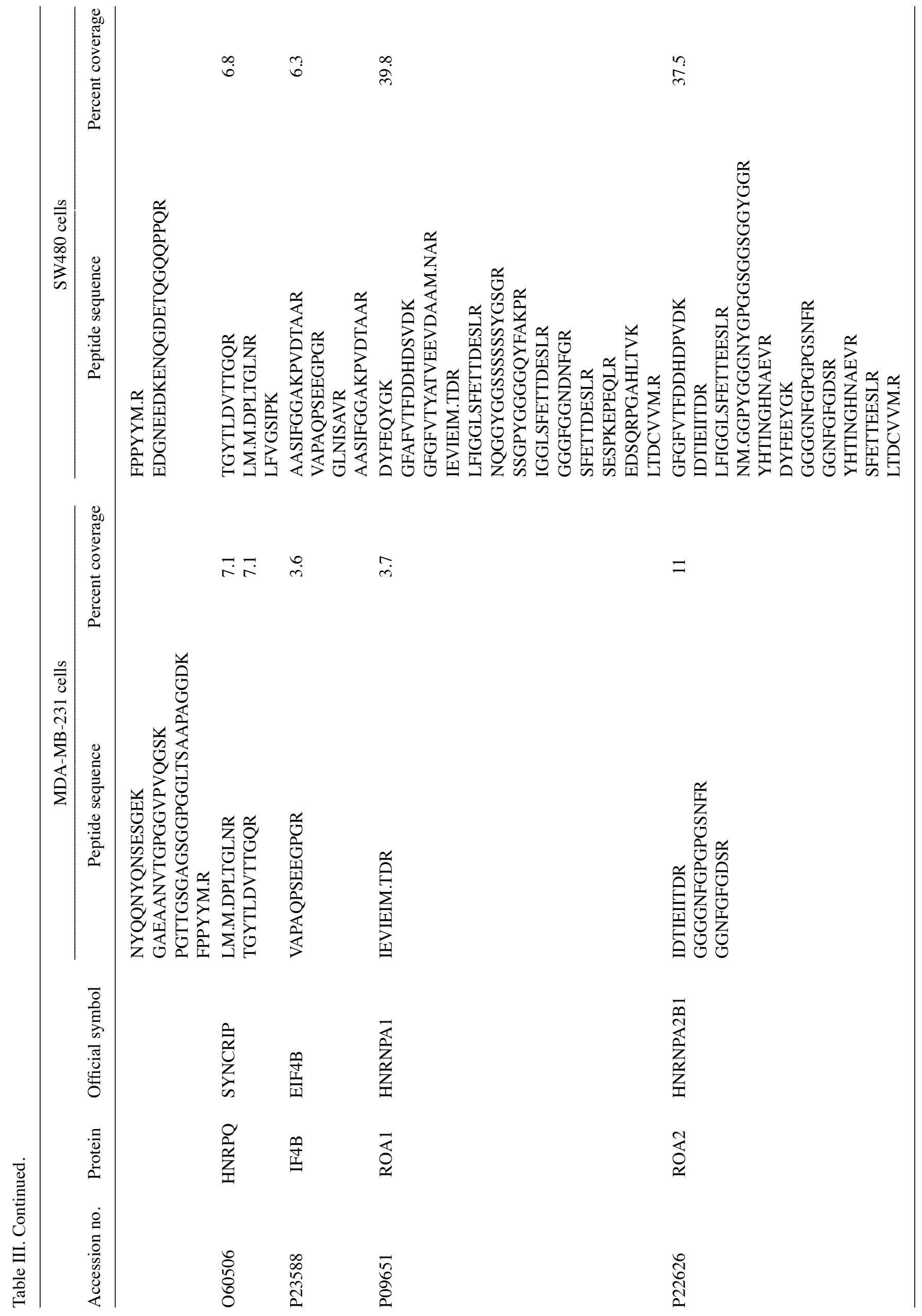


esis programme in somatic cells; this programme might contribute characteristic features to the neoplastic phenotype, including immortality, invasiveness, hypomethylation and metastatic capacity (42). Costa et al (43) believed that $\mathrm{CT}$ genes play a role in stem cell self-renewal; the expression of CT genes in tumor tissues contributes to maintaining stem cell properties and favors tumor proliferation. This hypothesis was supported by Yamada et al (44) who found that considerable numbers of CT genes showed preferential expression in cancer stem-like cells. Previous studies have shown that MAEL is necessary for proper germline stem cell lineage differentiation (11) and knockdown of MAEL expression disrupts the differentiation of mouse embryonic stem cells into germ cells (45). Therefore, whether MAEL also plays a role in cancer stem cells warrants further investigation.

In summary, we used proteomic approaches for isolating the interacting partners of MAEL, and demonstrated that MAEL, a component of nuage in germline cells, localizes in SGs and interacts with several components of SGs, suggesting that MAEL plays a role in carcinogenesis by post-transcriptionally regulating gene expression, as it does in gametogenesis. This finding should be valuable toward understanding the function of MAEL in carcinogenesis.

\section{Acknowledgements}

The present study was supported by the National Natural Science Foundation of China (grant nos. 81272318 and 81071656).

\section{References}

1. Clegg NJ, Frost DM, Larkin MK, Subrahmanyan L, Bryant Z and Ruohola-Baker H: maelstrom is required for an early step in the establishment of Drosophila oocyte polarity: posterior localization of rk mRNA. Development 124: 4661-4671, 1997.

2. Clegg NJ, Findley SD, Mahowald AP and Ruohola-Baker H: Maelstrom is required to position the MTOC in stage 2-6 Drosophila oocytes. Dev Genes Evol 211: 44-48, 2001.

3. Findley SD, Tamanaha M, Clegg NJ and Ruohola-Baker H: Maelstrom, a Drosophila spindle-class gene, encodes a protein that colocalizes with Vasa and RDE1/AGO1 homolog, Aubergine, in nuage. Development 130: 859-871, 2003.

4. Pek JW, Patil VS and Kai T: piRNA pathway and the potential processing site, the nuage, in the Drosophila germline. Dev Growth Differ 54: 66-77, 2012.

5. Lim AK and Kai T: Unique germ-line organelle, nuage, functions to repress selfish genetic elements in Drosophila melanogaster. Proc Natl Acad Sci USA 104: 6714-6719, 2007.

6. Costa Y, Speed RM, Gautier P, et al: Mouse MAELSTROM: the link between meiotic silencing of unsynapsed chromatin and microRNA pathway? Hum Mol Genet 15: 2324-2334, 2006.

7. Soper SF, van der Heijden GW, Hardiman TC, et al: Mouse maelstrom, a component of nuage, is essential for spermatogenesis and transposon repression in meiosis. Dev Cell 15: 285-297, 2008.

8. Aravin AA, van der Heijden GW, Castaneda J, Vagin VV, Hannon GJ and Bortvin A: Cytoplasmic compartmentalization of the fetal piRNA pathway in mice. PLoS Genet 5: e1000764, 2009.

9. Yokota S: Nuage proteins: their localization in subcellular structures of spermatogenic cells as revealed by immunoelectron microscopy. Histochem Cell Biol 138: 1-11, 2012.

10. Takebe M, Onohara Y and Yokota S: Expression of MAEL in nuage and non-nuage compartments of rat spermatogenic cells and colocalization with DDX4, DDX25 and MIWI. Histochem Cell Biol 140: 169-181, 2013
11. Pek JW, Lim AK and Kai T: Drosophila maelstrom ensures proper germline stem cell lineage differentiation by repressing microRNA-7. Dev Cell 17: 417-424, 2009.

12. Xiao L, Wang Y, Zhou Y, et al: Identification of a novel human cancer/testis gene MAEL that is regulated by DNA methylation. Mol Biol Rep 37: 2355-2360, 2010.

13. Janic A, Mendizabal L, Llamazares S, Rossell D and Gonzalez C: Ectopic expression of germline genes drives malignant brain tumor growth in Drosophila. Science 330: 1824-1827, 2010.

14. Hofmann O, Caballero OL, Stevenson BJ, et al: Genome-wide analysis of cancer/testis gene expression. Proc Natl Acad Sci USA 105: 20422-20427, 2008.

15. De Smet $\mathrm{C}$ and Loriot A: DNA hypomethylation and activation of germline-specific genes in cancer. Adv Exp Med Biol 754: 149-166, 2013.

16. Kim R, Kulkarni P and Hannenhalli S: Derepression of cancer/ testis antigens in cancer is associated with distinct patterns of DNA hypomethylation. BMC Cancer 13: 144, 2013.

17. Kim YH, Lee HC, Kim SY, et al: Epigenomic analysis of aberrantly methylated genes in colorectal cancer identifies genes commonly affected by epigenetic alterations. Ann Surg Oncol 18: 2338-2347, 2011.

18. Landthaler M, Gaidatzis D, Rothballer A, et al: Molecular characterization of human Argonaute-containing ribonucleoprotein complexes and their bound target mRNAs. RNA 14: 2580-2596, 2008.

19. Lin Z, Crockett DK, Lim MS and Elenitoba-Johnson KS: High-throughput analysis of protein/peptide complexes by immunoprecipitation and automated LC-MS/MS. J Biomol Tech 14: 149-155, 2003.

20. Keller A, Eng J, Zhang N, Li XJ and Aebersold R: A uniform proteomics MS/MS analysis platform utilizing open XML file formats. Mol Syst Biol 1: 2005.0017, 2005.

21. Keller A, Nesvizhskii AI, Kolker E and Aebersold R: Empirical statistical model to estimate the accuracy of peptide identifications made by MS/MS and database search. Anal Chem 74: 5383-5392, 2002.

22. Nesvizhskii AI, Keller A, Kolker E and Aebersold R: A statistical model for identifying proteins by tandem mass spectrometry. Anal Chem 75: 4646-4658, 2003.

23. Huang da W, Sherman BT and Lempicki RA: Systematic and integrative analysis of large gene lists using DAVID bioinformatics resources. Nat Protoc 4: 44-57, 2009.

24. Zhou J, Qiao X, Xiao L, et al: Identification and characterization of the novel protein CCDC106 that interacts with p53 and promotes its degradation. FEBS Lett 584: 1085-1090, 2010.

25. Kedersha N, Stoecklin G, Ayodele M, et al: Stress granules and processing bodies are dynamically linked sites of mRNP remodeling. J Cell Biol 169: 871-884, 2005.

26. Rothe F, Gueydan C, Bellefroid E, Huez G and Kruys V: Identification of FUSE-binding proteins as interacting partners of TIA proteins. Biochem Biophys Res Commun 343: 57-68, 2006.

27. Onishi H, Kino Y, Morita T, Futai E, Sasagawa N and Ishiura S: MBNL1 associates with YB-1 in cytoplasmic stress granules. J Neurosci Res 86: 1994-2002, 2008.

28. Quaresma AJ, Bressan GC, Gava LM, Lanza DC, Ramos CH and Kobarg J: Human hnRNP Q re-localizes to cytoplasmic granules upon PMA, thapsigargin, arsenite and heat-shock treatments. Exp Cell Res 315: 968-980, 2009.

29. Wen F, Zhou R, Shen A, Choi A, Uribe D and Shi J: The tumor suppressive role of eIF3f and its function in translation inhibition and rRNA degradation. PLoS One 7: e34194, 2012.

30. Wolf A, Krause-Gruszczynska M, Birkenmeier O, OstareckLederer A, Huttelmaier S and Hatzfeld M: Plakophilin 1 stimulates translation by promoting eIF4A1 activity. J Cell Biol 188: 463-471, 2010.

31. Buchan JR, Yoon JH and Parker R: Stress-specific composition, assembly and kinetics of stress granules in Saccharomyces cerevisiae. J Cell Sci 124: 228-239, 2011.

32. Gallouzi IE, Brennan CM, Stenberg MG, et al: HuR binding to cytoplasmic mRNA is perturbed by heat shock. Proc Natl Acad Sci USA 97: 3073-3078, 2000.

33. Guil S, Long JC and Caceres JF: hnRNP A1 relocalization to the stress granules reflects a role in the stress response. Mol Cell Biol 26: 5744-5758, 2006.

34. Kim HJ, Kim NC, Wang YD, et al: Mutations in prion-like domains in hnRNPA2B1 and hnRNPA1 cause multisystem proteinopathy and ALS. Nature 495: 467-473, 2013. 
35. Shih JW, Wang WT, Tsai TY, Kuo CY, Li HK and Wu Lee YH: Critical roles of RNA helicase DDX3 and its interactions with eIF4E/PABP1 in stress granule assembly and stress response. Biochem J 441: 119-129, 2012.

36. Goodier JL, Zhang L, Vetter MR and Kazazian $\mathrm{HH} \mathrm{Jr}$ : LINE-1 ORF1 protein localizes in stress granules with other RNA-binding proteins, including components of RNA interference RNA-induced silencing complex. Mol Cell Biol 27: 6469-6483, 2007.

37. Wippich F, Bodenmiller B, Trajkovska MG, Wanka S, Aebersold R and Pelkmans L: Dual specificity kinase DYRK3 couples stress granule condensation/dissolution to mTORC1 signaling. Cell 152: 791-805, 2013.

38. Anderson P and Kedersha N: Stress granules: the Tao of RNA triage. Trends Biochem Sci 33: 141-150, 2008.

39. Decker CJ and Parker R: P-bodies and stress granules: possible roles in the control of translation and mRNA degradation. Cold Spring Harb Perspect Biol 4: a012286, 2012.

40. Anderson $P$ and Kedersha N: RNA granules: post-transcriptional and epigenetic modulators of gene expression. Nat Rev Mol Cell Biol 10: 430-436, 2009.
41. Gjerstorff MF, Rosner HI, Pedersen CB, et al: GAGE cancergermline antigens are recruited to the nuclear envelope by germ cell-less (GCL). PLoS One 7: e45819, 2012.

42. Simpson AJ, Caballero OL, Jungbluth A, Chen YT and Old LJ: Cancer/testis antigens, gametogenesis and cancer. Nat Rev Cancer 5: 615-625, 2005.

43. Costa FF, Le Blanc K and Brodin B: Concise review: cancer/ testis antigens, stem cells, and cancer. Stem Cells 25: 707-711, 2007.

44. Yamada R, Takahashi A, Torigoe T, et al: Preferential expression of cancer/testis genes in cancer stem-like cells: proposal of a novel sub-category, cancer/testis/stem gene. Tissue Antigens 81: 428-434, 2013.

45. Bahena I, Xu E, Betancourt M, et al: Role of Mael in early oogenesis and during germ-cell differentiation from embryonic stem cells in mice in vitro. Zygote 1-8, 2013 [Epub ahead of print]. 